Documentation et bibliothèques

DOCUMENTATION BIBLIOTHEQUES

\title{
La planification nationale des services d'information documentaire
}

\section{Réal Bosa}

Volume 24, numéro 3, septembre 1978

URI : https://id.erudit.org/iderudit/1055164ar

DOI : https://doi.org/10.7202/1055164ar

Aller au sommaire du numéro

\section{Éditeur(s)}

Association pour l'avancement des sciences et des techniques de la documentation (ASTED)

\section{ISSN}

0315-2340 (imprimé)

2291-8949 (numérique)

Découvrir la revue

Citer cet article

Bosa, R. (1978). La planification nationale des services d'information documentaire. Documentation et bibliothèques, 24(3), 129-135.

https://doi.org/10.7202/1055164ar

\section{Résumé de l'article}

L'évolution des services de bibliothèques et de documentation, tant dans les pays développés que dans les pays en développement, a conduit ces derniers à modifier, et cela globalement, leur approche en matière de développement. Plusieurs raisons militent en faveur d'une planification à l'échelon national des services documentaires : l'inflation monétaire, l'explosion de l'information et les moyens requis (tels les systèmes et réseaux) pour en assurer, grâce à l'évolution des technologies, un meilleur contrôle et une plus large utilisation. Un certain nombre de gouvernements se sont déjà résolument engagés dans le sens d'une planification nationale. Cette dernière implique, entre autres, la reconnaissance officielle de l'information comme facteur primordial de développement, l'inventaire des ressources existantes ainsi que des études sur leur utilisation, l'établissement d'un réseau national et l'élaboration de programmes de mise en valeur des ressources documentaires.
Tous droits réservés (c) Association pour l'avancement des sciences et des techniques de la documentation (ASTED), 1978
Ce document est protégé par la loi sur le droit d'auteur. L'utilisation des services d'Érudit (y compris la reproduction) est assujettie à sa politique d'utilisation que vous pouvez consulter en ligne.

https://apropos.erudit.org/fr/usagers/politique-dutilisation/ 


\title{
La planification nationale des services d'information documentaire
}

\author{
Réal Bosa \\ Bibliothèque nationale du Québec \\ Montréal
}

L'évolution des services de bibliothèques et de documentation, tant dans les pays développés que dans les pays en développement, a conduit ces derniers à modifier, et cela globalement, leur approche en matière de développement. Plusieurs raisons militent en faveur d'une planification à l'échelon national des services documentaires: l'inflation monétaire, l'explosion de l'information et les moyens requis (tels les systèmes et réseaux) pour en assurer, grâce à l'évolution des technologies, un meilleur contrôle et une plus large utilisation. Un certain nombre de gouvernements se sont déjà résolument engagés dans le sens d'une planification nationale. Cette dernière implique, entre autres, la reconnaissance officielle de l'information comme facteur primordial de développement, l'inventaire des ressources existantes ainsi que des études sur leur utilisation, l'établissement d'un réseau national et l'élaboration de programmes de mise en valeur des ressources documentaires.

The evolution of library and documentation services in developed countries, as well as in underdeveloped countries, has led the latter to modify totally their approach to development. Several reasons justify national planning of documentary resources: monetary inflation, the explosion of information and the means required, (such as systems and networks) to insure through the evolving technology, its better control and a wider use. A certain number of governments have already committed themselves to national planning. This implies an official recognition of information the main as factor in development, the inventory of existing resources as well as studies on their use, the establishment of a national network and the creation of programmes to promote documentary resources.

La evolución de los servicios de bibliotecas y de documentación, en los países desarrollados como en los países en vía de desarrollo, llevó estos paises a modificar globalmente su aproximiento al desarrollo. Existen motivos en favor de una planificación nacional de los servicios de documentación: la inflación monetaria, la explosión de la información y los medios necesarios (como los sistemas y las redes) para asegurar, gracias a la evolución de las tecnologías, un mejor control y una utilización más grande. Algunos gobiernos han entrado ya en el proceso de planificación nacional. Ésto implica, entre otras cosas, el reconozimiento oficial de la información como factor esencial de desarrollo, el inventario de los recursos existentes asi como estudios sobre su utilización, el establecimiento de una red nacional y la elaboración de programas para poner de manifiesto los recursos documentales.

N.D.L.R. - Ce texte de Réal Bosa s'inscrit dans le cadre plus général d'un document de travail remis aux participants d'une Table ronde qui s'est tenue à Québec, le 4 mai 1978, sous le thème suivant: La Planification des services documentaires québécois.

Cette Table ronde était organisée par l'ASTED (Association pour l'avancement des sciences et des techniques de la documentation ) et avait pour objectif principal "d'obtenir des instances politiques qu'elles prennent l'initiative d'établir, à brève échéance, une politique et des mécanismes pour une planification québécoise des services documentaires ". 
Elle regroupait des ministres du Gouvernement du Québec dont $M$. Camille Laurin et M. Louis O'Neil ou des représentants des cinq ministères les plus concernés par le sujet, quelques experts internationaux: Carlos Victor Penna, ex-Directeur de la Division des bibliothèques de la documentation et des archives (UNESCO), Morris Matza, secrétaire exécutif de Systedma nacional informacion (Venezuela) et Alphonse Trezza, Directeur général de la National Commission on Libraries and Information Science (NACLIS ) U.S.A., ainsi que quelques spécialistes québécois du milieu de la bibliothéconomie et de la documentation.

L'importance majeure que l'on accorde à l'information comme facteur de progrès des sociétés contemporaines et l'évolution vertigineuse que cette dernière connaît à la fois dans ses dimensions, ses formes et ses modes de transmission ont conduit, au cours des dernières décennies, les services de bibliothèques et de documentation dans le monde à explorer des avenues nouvelles dans leur fonctionnement et même, dans certains cas, à modifier de façon radicale les structures organisationnelles qui les régissent.

C'est ainsi que s'est développée, et cela sur une large échelle au niveau des pays dits développés, la coopération entre bibliothèques d'une même catégorie ou d'un milieu géographique donné. Ce concept, largement exploré par le passé dans la littérature bibliothéconomique, s'est enfin traduit dans des réalités bien concrètes s'appliquant soit à l'acquisition, soit au traitement ou à l'accessibilité, bibliographique et physique, des collections. Des pays ou ensembles nationaux conçurent ainsi des projets de grande envergure, tels le Scandia Plan, le Farmington Plan, qui, outre le mérite d'exister, ont permis de résoudre dans l'immédiat un certain nombre de problèmes et surtout de développer le sens de l'interdépendance des bibliothèques en matière de ressources documentaires. L'objectif plus ou moins avoué de ces initiatives consistait à mettre à l'épreuve les structures existantes et à vérifier leur capacité véritable d'adaptation au phénomène nouveau de l'envahissement de l'information.

Au cours de cette même période, les pays en voie de développement, contraints de faire face aux mêmes défis et, en plus, de combler d'immenses retards en matière d'organisation des services de bibliothèques et de documentation, exploraient des approches nouvelles mieux adaptées à leur contexte particulier.
Ainsi s'est élaboré, grâce à la collaboration des dirigeants nationaux, des spécialistes locaux et surtout avec l'immense concours des experts étrangers et des organisations internationales, le concept de la planification nationale des services de bibliothèques et de documentation conçue comme partie intégrante du développement global d'un pays. Ce concept, représentant une approche inédite jusque-là ( sauf peut-être en Union soviétique), allait par la suite être approfondi grâce à des études publiées par des spécialistes ${ }^{1}$ et à des réunions d'experts organisées par l'Unesco sur la planification nationale dans différentes régions du globe: Amérique latine, Asie, Afrique et pays arabes.

De ces deux démarches de pays et d'ensembles nationaux se situant à des niveaux opposés de développement, l'une centrée sur la "maximisation" de l'organisation existante par le biais de la coopération et l'autre tournée vers des modèles nouveaux, l'expérience acquise semble devoir déjà démontrer que la dernière est de beaucoup la plus prometteuse.

II est révélateur en effet que des pays aussi riches en ressources documentaires et en moyens financiers affectés à leur exploitation que les Etats-Unis et la Grande-Bretagne en soient venus à devoir modifier, et cela globalement, leur approche en matière de développement des servíces de bibliothèques et de documentation. A cet égard, le constat établi par la National Commission on Libraries and Information Science (NCLIS) américaine, dans son étude Toward a National Program for Library and Information Services: Goals for Action, est éloquent:

\footnotetext{
1. Par exemple, C.V. Penna, La planification des services de bibliothèques et de documentation, $2 \mathrm{e}$ éd. rev. et augm., Paris, Unesco, 1971.
} 
"The growth of libraries in the United States has been fragmentary and uneven. They evolved independently and do not presently constitute an orderly national system.

Library and other information resources in the United States are unevenly distributed, a fact which stems from the uneven population distribution and diverse tax structures in the country.

There is a critical need to identify and address the problems of those without even the most basic information services and those who are being served only marginally.

Special libraries with information resources for work-related needs exist throughout the United States, but at present they serve only a limited clientele.

Funding for most library and information services at every level - local, state, multistate, and Federal - is inadequate.

New Federal and state legislation is needed which will give local libraries the incentive activity to join larger systems of service outside their immediate jurisdictions. ${ }^{2}$

L'expérience américaine est instructive d'autant plus qu'aucun autre pays n'a probablement exploré autant que les Etats-Unis des formes inédites et institutionnalisées de coopération inter-bibliothèques faisant appel à de nouvelles technologies, ni autant investi en matière de ressources documentaires.

Le cas québécois est également éloquent à sa manière. Considéré dans son ensemble, le développement des bibliothèques au Québec s'est effectué très largement selon l'approche organisationnelle en vigueur au Canada et aux Etats-Unis, i.e. par un cloisonnement des bibliothèques, tant au niveau gouvernemental que local. Bien que des crédits importants aient été consentis par l'Etat, il est devenu

2. National Commission on Libraries and Information Science, Toward a National Program for Library and Information Services: Goals for Action, Washington, D.C., 1975 , p. 23-24 rapidement évident que les services de bibliothèques et de documentation, fonctionnant isolément, se révélaient incapables de répondre aux besoins nouveaux correspondant à l'évolution de la société québécoise. La voie était donc ouverte à la coopération interbibliothèques. Or, il ressort clairement des nombreuses discussions entre professionnels québécois et des quelques initiatives sectorielles conduites jusqu'ici que le chemin vers une coopération structurée, s'inspirant du modèle américain, sera long, les orientations actuelles rendant la marche inutile et la voie sans issue. Le président de l'ASTED avouait lui-même en 1977 :

"ll nous semble malheureusement clair que les spécialistes en information documentaire ne pourront pas régler, par eux-mêmes, le problème du cloisonnement documentaire qui les affecte. Nous avons présenté trop de mémoires et trop de rapports pour pouvoir encore rêver de faire triompher notre point de vue. A notre avis, nous devrons nous résigner à l'idée que la consolidation viendra du sommet ou qu'elle ne se fera pas. ${ }^{3}$

\section{Les fondements d'une planification nationale}

On a pu constater, jusqu'à une période assez récente, que le secteur des bibliothèques et des services de documentation opérait en vase clos, régi par des règles établies par ses spécialistes à la lumière de ce que C.V. Penna qualifie de "contemporary professional discussion and philosophy $\gg 4$. Cette situation se reflétait et dans la littérature bibliothéconomique qui ne se renouvelait guère, et dans l'activité professionnelle en général qui s'exerçait selon des schèmes devenus traditionnels.

La montée au début de la présente décennie d'une conjoncture économique plus fragile n'allait pas tarder à affecter les divers secteurs de l'activité nationale et à manifester ses

3. Daniel Reicher, "Vers une consolidation des services québécois d'information documentaire ", Documentation et bibliothèques, vol. 23, no 3 (septembre 1977), 126.

4. C.V. Penna et al., National Library and Information Services; a Handbook for Planners, London, Butterworths, 1977, p. 1. 
effets au plan du fonctionnement et du développement général des bibliothèques et services de documentation. C'est ainsi que l'on a pu constater (et la situation s'est aggravée depuis ) l'apparition d'une disproportion inquiétante au niveau des budgets entre les sommes affectées à l'acquisition de la documentation et les salaires dépensés pour la conserver, la traiter et l'exploiter, et une baisse considérable du nombre de documents acquis, compte tenu de l'augmentation exorbitante du coût des livres et des périodiques. Par ailleurs, les gouvernements se sont vus contraints de freiner sensiblement le développement des bibliothèques, contribuant ainsi à créer une nouvelle catégorie de professionnels chômeurs. On a pu constater également que des décisions malheureuses ont été prises dans certains pays visant à remplacer les professionnels par un personnel de moindre compétence à la direction des services documentaires.

Ce contexte nouveau d'une économie de moyens posait des dilemmes qui en soi n'étaient pas insolubles, n'eût été l'apparition d'un deuxième phénomène, celui de l'explosion documentaire.

Selon une étude publiée en 1973 par l'OCDE 5 , la quantité d'information produite dans le monde augmente à un rythme qui la fait doubler tous les cinq ans; cela signifie que, de 1970 à 1985, elle aura augmenté de six cents pour cent. Or l'accès à cette masse documentaire s'avère indispensable à une époque fortement dominée par le développement technologique, où la recherche, tant celle des individus que des institutions, est devenue facteur primordial de développement économique et social. D'autre part, une scolarisation plus élevée et l'accroissement des programmes de formation permanente ont développé, tant chez le simple citoyen que chez l'ouvrier spécialisé, le professionnel et l'étudiant, des besoins nouveaux en information que les bibliothèques scolaires, publiques ou spécialisées réussissent de moins en moins à combler.

Des méthodes nouvelles ont été explorées ces dernières années en vue d'assurer le

5. Georges Anderla, L'information en 1985; une étude prévisionnelle des besoins et des ressources, Paris, OCDE, 1973, p. 65. harnachement de ce flot documentaire. C'est ainsi que sont nés et se sont développés à l'échelon national et international des réseaux et systèmes appelés à gérer et à diffuser l'information dans les domaines scientifiques et techniques, de même que pour les sciences de l'homme. Nous citons ici à titre d'exemples certains de ces systèmes établis à l'échelle mondiale: AGRIS, DEVSIS, UNISIST, le CBU6. Ces systèmes mondiaux, de même que les réseaux établis sur une base nationale ou régionale, font appel à l'usage de nouvelles technologies et, à ce titre, posent de nouveaux défis aux services de bibliothèques et de documentation, organisés jusqu'ici selon des schèmes plutôt traditionnels. Ces défis relèvent de l'ordre des compétences requises, mais également des moyens financiers que les services actuels, considérés globalement, ne peuvent assumer. Cet impact de la technologie a été largement évoqué, d'ailleurs, comme justificatif d'une planification nationale par les participants à la Conférence intergouvernementale organisée par l'Unesco en 1974:

"D'abord la technologie est coûteuse et le gouvernement doit dès le début s'engager à long terme pour assurer la stabilité du programme. En second lieu, la technologie étant complexe, il est indispensable qu'il existe au niveau national une direction technique éclairée si l'on veut que toutes les institutions intéressées coordonnent efficacement leurs activités. En troisième lieu, la technologie est spécialisée et l'évolution d'un programme national d'information doit être synchronisée avec l'enseignement technique dispensé aux personnes qui en assureront l'application. Enfin, en raison du caractère novateur de la technologie, son introduction modifie invariablement les habitudes et exige que l'on se penche sur les problèmes de recyclage de l'utilisateur. »7

6. AGRIS, DEVSIS et UNISIST constituent des systèmes mondiaux d'information respectivement dans les secteurs de l'agriculture, du développement, des sciences et de la technologie. Le CBU (Contrôle bibliographique universel) vise l'accessibilité générale à l'information bibliographique.

7. Conférence intergouvernementale sur la planification des infrastructures nationales en matière de documentation, de bibliothèques et d'archives, Paris, 23-27 septembre 1974, Rapport final, Paris, Unesco, 1974 , p. 31 
II est devenu évident que le poids et l'interaction des facteurs nouveaux évoqués plus haut, tels l'inflation monétaire, l'explosion de l'information et les moyens requis pour en assurer, grâce à l'évolution des technologies, un meilleur contrôle et une plus large utilisation, auront tôt fait d'élargir le fossé entre l'information existante et celle effectivement mise à la disposition des utilisateurs dans nos bibliothèques et services de documentation; à moyen terme, ils relégueront aux oubliettes nos institutions pourtant vénérables si des actions énergiques ne sont pas entreprises dans les meilleurs délais au plan national.

\section{Les engagements nationaux}

L'état d'urgence évoqué tant par les utilisateurs que par les milieux de la documentation aura contribué à sensibiliser comme jamais auparavant les dirigeants politiques à la nécessité d'une intervention énergique dans le sens d'une planification nationale.

Un certain nombre de gouvernements, dont certains peu taxables d'interventionnisme dans les domaines de l'éducation et de la culture, ont entrepris récemment de poser des actes concrets dans ce sens. C'est le cas notamment des Etats-Unis, de la Grande-Bretagne, du Venezuela, de la Jamaïque, de la République fédérale d'Allemagne, du Ghana, des pays scandinaves.

Ces gouvernements, il convient de le dire, ont fait preuve d'audace, ne pouvant bénéficier d'aucune expérience similaire entreprise au plan national, sauf peut-être de celle vécue en Union soviétique. Ils ont pu s'appuyer cependant sur un certain nombre d'études conduites par des spécialistes et s'inspirer des expériences dans des domaines voisins, tel le secteur de l'éducation.

Par l'organisation de rencontres régionales et grâce à l'expertise acquise à la fois par ses responsables en matière de bibliothèques et ses consultants dans les pays en voie de développement, l'Unesco aura assumé dans ce secteur un leadership certain. C'est à cet organisme que revient l'initiative de l'organisation à Paris en 1974 de la Conférence intergouvernementale sur la planification des infrastructures nationales en matière de documentation, de bibliothèques et d'archives, conférence dont l'aboutissement aura été l'adoption par tous les pays membres du concept général d'une planification globale des infrastructures nationales formulé dans un document intitulé Systèmes nationaux d'information (NATIS): objectifs d'une action nationale et internationale.

L'Unesco a choisi d'investir des sommes considérables au cours des prochaines années, en vue de mener les études requises, d'aider les pays membres et de favoriser les rencontres d'experts sur cette question.

Les gouvernements engagés dans cette expérience de planification et ceux qui ont entrepris de s'y impliquer ont tout intérêt à bénéficier de cette aide, de même qu'à faire partager à la communauté internationale, par le biais d'une large information, leurs expériences en ce domaine. L'enrichissement en serait d'autant plus grand que les approches sont et seront nécessairement diversifiées, compte tenu des divers contextes nationaux dans lesquels elles s'insèrent.

\section{Lignes directrices en matière de planification nationale}

L'intervention gouvernementale dans le domaine de la planification nationale des services de bibliothèques et de documentation est fondée essentiellement sur la reconnaissance de l'information comme facteur primordial du développement national et sur l'obligation, largement reconnue par les Etats dans le monde, d'assurer à tous les citoyens, indépendamment de leur condition sociale ou de leur milieu géographique, le plein exercice du droit à l'information.

Cette double reconnaissance, que les Etats devraient traduire dans des textes officiels, constitue le fondement essentiel, l'éclairage d'ensemble qui doit sous-tendre tous les efforts à entreprendre aux divers paliers d'intervention.

Dans une telle perspective, les ressources documentaires dispersées sur le territoire de la nation se conçoivent aisément comme constituant des ressources dites nationales, d'une importance aussi névralgique que certaines 
ressources naturelles, tels que l'eau et le pétrole.

Une telle perception ne relève pas d'un souci de collectivisme à outrance, mais d'un sain réalisme, si l'on tient compte de la valeur intrinsèque de ces ressources et des investissements que représentent aujourd'hui leur acquisition, leur traitement et leur diffusion. Cette perception, des auteurs éminents l'ont formulée en des termes non équivoques:

"No society can advance beyond a certain point without effective access to its collective memory of record, or conversely, an advanced society that loses control of the record will regress. $\gg 8$ (R.C. Swank ).

"La science a atteint un tel degré d'unité et de complexité que les services de bibliothèques et d'information sont devenus la clé de tout progrès conscient sur tout le front du savoir en marche. $\gg 9$

L'inventaire des ressources documentaires existantes, tant dans le secteur public que privé, devient dans ce contexte d'une grande importance en vue de déterminer leur dimension et leur niveau, de même que leur répartition géographique.

Cet inventaire doit s'accompagner théoriquement d'une étude, à l'échelon national, portant sur l'utilisation de ces ressources par les usagers. Les conclusions de travaux de ce genre, entrepris dans divers pays, sur des bases sectorielles, ont conduit, on le sait, nombre de bibliothèques à modifier leurs politiques en matière de développement des collections.

Cette démarche de l'analyse des ressources, jointe à une étude sur leur utilisation, a conduit les pays qui l'ont entreprise ( même sur une base sectorielle ) à concevoir des réseaux d'information documentaire dont l'objectif consiste essentiellement à favoriser, grâce à l'usage des nouvelles technologies, un accès

8. R.C. Swank, «Interlibrary cooperation, interlibrary communications, and information networks explanation and definition", in Proceedings of the Conference on Interlibrary Communications and Information Networks, Chicago, ALA, 1971, p. 20.

9. Citation de l'éminent physicien Bernal, 1945. plus large à l'information et, par voie de conséquence, à assurer une meilleure rentabilité des investissements en matière documentaire. De tels réseaux existent dans bon nombre de pays. Cependant, ils sont constitués soit de bibliothèques de mêmes catégories, soit d'établissements d'une région géographique donnée et ils contribuent ainsi à accentuer les disparités régionales et à restreindre à certaines catégories d'usagers le plein usage du droit à l'information.

L'établissement d'un réseau national d'information documentaire représente une étape majeure dans la planification nationale. Cette étape doit précéder, ou tout au moins accompagner l'injection massive de fonds en vue d'accroitre les ressources documentaires dans les secteurs jugés plus faibles. Les investissements financiers de l'Etat dans ce domaine se révéleront cependant importants à court terme, compte tenu des implications en matière de normalisation, d'appareillage technologique et de formation du personnel.

La création d'un tel réseau, en plus de favoriser un accès rapide et plus généralisé à l'information, conduira forcément les bibliothèques et les services de documentation à modifier de façon sensible leurs politiques et leurs procédures, réduisant d'autant la part d'intervention des gouvernements.

Nous voudrions évoquer enfin un dernier aspect de la question, généralement négligé, celui de la promotion de la lecture. L'importance des investissements de l'Etat en vue d'assurer plus largement le droit à l'information dans une société dite "éducative» démontre clairement la nécessité pour ce dernier de planifier les moyens requis visant à assurer que la recherche de l'information devienne un réflexe normal dans la vie quotidienne des citoyens et que les usagers en général soient initiés à une meilleure utilisation des services documentaires. Deux objectifs assignés au NATIS, "Stimuler la prise de conscience de l'usager » et "Encourager l'habitude de lire", dénotent l'importance d'une telle action entreprise à l'échelon national.

\section{Conclusion}

Dans son allocution à la séance d'ouverture de la Conférence intergouvernementale sur la 
planification des bibliothèques tenue en 1974, le directeur général de l'Unesco, René Maheu, après avoir évoqué l'ampleur et la complexité extrêmes des problèmes que pose l'information en cette seconde moitié du XXe siècle, invitait les états membres à réaliser

"ce grand dessein... qui n'est rien moins que la recherche, à l'échelon national et international, des solutions nouvelles et imaginatives aux problèmes relatifs au stockage, à la mobilité et à la communication de l'information sous toutes ses formes et dans tous les domaines qu'appelle le progrès de notre civilisation. $\gg 10$

10. Conférence intergouvernementale..., 43.
Dans cette perspective, et compte tenu des retards accumulés en matière de développement des ressources documentaires, le dessein québécois peut être jugé ambitieux, si l'on prend en considération les investissements qui seront requis à moyen terme en vue d'instaurer un réseau national d'information. Nous croyons cependant qu'il est réalisable sur la base d'une pleine concertation de l'Etat et des milieux professionnels. De plus, il s'inscrit tout à fait, selon nous, dans les priorités du gouvernement québécois en matière d'utilisation rationnelle des ressources, tant matérielles qu'humaines, et de développement sur son territoire d'une identité culturelle distincte, mieux assurée par un large accès de tous ses citoyens à l'information.

\section{NOUVEAUTÉS}

Mélanges d'histoire du Canada français offerts au professeur Marcel Trudel $17 \times 25 \mathrm{~cm}, 256$ pages. Prix: $\$ 9.00$

Le système monétaire international en voie de réforme

Les travaux du Comité des Vingt par Duncan Cameron

$15 \times 23 \mathrm{~cm}, 190$ pages. Prix: $\$ 6.00$

\section{Lettres de Margry à Parkman 1872-1892}

par Louis-Philippe Cormier

$15 \times 23 \mathrm{~cm}, 220$ pages. Prix: $\$ \mathbf{1 0 . 0 0}$

Langue et religion

Histoire des conflits anglo-français en Ontario

par Robert Choquette

$15 \times 23 \mathrm{~cm}, 268$ pages. Prix: $\$ 7.50$

En vente chez votre libraire et aux:

\section{Editions de l'Université d'Ottawa \\ 65, avenue Hastey \\ Ottawa (Ont.) \\ K1N 6N5}

